\title{
A PRIORI BOUNDS FOR POSITIVE SOLUTIONS OF A SEMILINEAR ELLIPTIC EQUATION
}

\author{
CHRIS COSNER AND KLAUS SCHMITT ${ }^{1}$
}

\begin{abstract}
We consider the semilinear elliptic equation $-\Delta u=f(u), x \in \Omega$, subject to zero Dirichlet boundary conditions, where $\Omega \subset \mathbf{R}^{n}$ is a bounded domain with smooth boundary and the nonlinearity $f$ assumes both positive and negative values. Under the assumption that $\Omega$ satisfies certain symmetry conditions we establish two results providing lower bounds on the $C^{0}(\bar{\Omega})$ norm of positive solutions. The bounds derived are the same one obtains in dimension $n=1$.
\end{abstract}

1. Introduction. In this note we establish lower bounds on the $C^{0}(\bar{\Omega})$ norm for certain positive solutions of the semilinear boundary value problem

$$
\begin{aligned}
\Delta u+f(u) & =0, & & x \in \Omega, \\
u & =0, & & x \in \partial \Omega,
\end{aligned}
$$

where $\Omega$ is a bounded domain in $\mathbf{R}^{n}$ with sufficiently smooth boundary and satisfying certain symmetry conditions. ${ }^{1}$

The bounds derived here are motivated by results derived in $[\mathbf{7}, \mathbf{8}]$ concerning continua of positive solutions of the one parameter problem

$$
\begin{aligned}
\Delta u+\lambda f(u) & =0, & & x \in \Omega, \\
u & =0, & & x \in \partial \Omega,
\end{aligned}
$$

and its finite difference analogues. The results of $[\mathbf{1}, \mathbf{3}, \mathbf{5}, \mathbf{7}$ and $\mathbf{8}]$ when applied to (2) in the case of the model nonlinearity $f$ given in Figure 1 yield a solution diagram for positive solutions as given in Figure 2.

In the case of ordinary differential equations one may employ phase plane methods to establish sharper lower bounds on the norms of solutions on the continua $\Sigma_{1}$ and $\Sigma_{\infty}$, namely:

$$
\begin{aligned}
& \text { If }(u, \lambda) \in \Sigma_{1} \text {, then }\|u\|_{0}=\max _{x \in \Omega} u(x) \geq r_{1} \text {, where } r_{1} \in\left(s_{1}, s_{2}\right) \\
& \text { is given by } \int_{s_{0}}^{r_{1}} f(s) d s=0 . \\
& \text { If }(u, \lambda) \in \Sigma_{\infty} \text {, then }\|u\|_{0} \geq r_{\infty} \text {, where } r_{\infty}>s_{3} \text { is given by } \\
& \int_{s_{2}}^{r_{\infty}} f(s) d s=0 .
\end{aligned}
$$

We shall establish the same results in higher dimensions, provided $\Omega$ satisfies the symmetry condition in [4].

We note here (see [7]) that the norm of positive solutions of corresponding finite difference approximation problems are not bounded away from $s_{3}$, respectively $s_{1}$,

Received by the editors September 17, 1984.

1980 Mathematics Subject Classification. Primary 35J25, 35J60, 34B15.

${ }^{1}$ Research supported by NSF grant MCS 8121951 . 


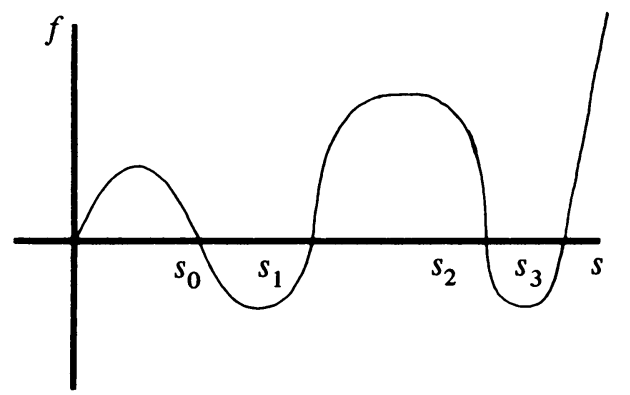

FIGURE 1

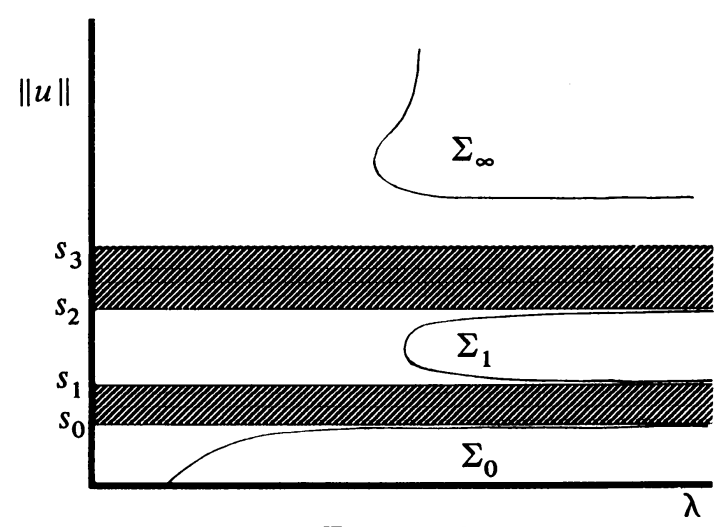

FIGURE 2

as $\lambda \rightarrow \infty$, and thus the solution branches of such approximating problems will not approximate the solution branches $\Sigma_{1}$ and $\Sigma_{\infty}$ in a neighborhood of $\lambda=\infty$.

2. Symmetry assumptions and consequences. In what is to follow we shall assume that $\Omega$ satisfies the following symmetry conditions:

(i) $0 \in \Omega$,

(ii) there exist $n$ linearly independent unit vectors $\gamma_{1}, \ldots, \gamma_{n}$ such that $\Omega$ is symmetric about each of the hyperplanes

$$
P\left(\gamma_{k}, 0\right)=\left\{x: x \cdot \gamma_{k}=0\right\}, \quad 1 \leq k \leq n,
$$

(iii) if $P\left(\gamma_{k}, \delta\right)=\left\{x: x \cdot \gamma_{k}=\delta\right\}$ and $\delta_{k}=\max \left\{|\delta|: P\left(\gamma_{k}, \delta\right) \cap \Omega \neq \varnothing\right\}$, then, for $0 \leq \delta<\delta_{k}$, the reflection of $\left\{x \in \Omega: x \cdot \gamma_{k}<\delta\right\}$ through $P\left(\gamma_{k}, \delta\right)$ lies in $\Omega$, and, similarly, for $-\delta_{k}<\delta \leq 0$, the reflection of $\left\{x \in \Omega: x \cdot \gamma_{k}<\delta\right\}$ through $P\left(\gamma_{k}, \delta\right)$ lies in $\Omega, 1 \leq k \leq n$.

(iv) the hyperplanes $P\left(\gamma_{k}, \delta\right), 0<|\delta|<\delta_{k}$, are not orthogonal to $\partial \Omega, 1 \leq k \leq n$.

These conditions imply the following result (see Theorem 2.1 of [4]):

THEOREM 1. Let $u \in C^{2}(\bar{\Omega})$ be a solution of (1) such that $u(x)>0, x \in \Omega$, and let $\Omega$ satisfy the above symmetry conditions. Then for all $x \in \Omega$ with $0<$ $x \cdot \gamma_{k}<\delta_{k}$ we have $\nabla u \cdot \gamma_{k}<0$, and, for all $x \in \Omega$ with $-\delta_{k}<x \cdot \gamma_{k}<0$, we have $\nabla u \cdot \gamma_{k}>0,1 \leq k \leq n$. In particular, $\nabla u(x)=0$ if and only if $x=0$. 
3. A priori bounds. The following assumptions will be imposed on $f$ :

(i) $f \in C^{1}[0, \infty)$,

(ii) $f(0) \geq 0$ and there exist $0<s_{0}<s_{1}<s_{2}$ such that $f\left(s_{1}\right)=0, f(s)>$ $0, s_{1}<s<s_{2}, f(s)<0, s_{0}<s<s_{1}, \int_{s_{0}}^{s_{2}} f(s) d s>0$.

THEOREM 2. Let $\Omega$ satisfy the above symmetry conditions with respect to $\gamma_{1}, \ldots, \gamma_{n}$, where $\gamma_{i}=e_{i}, 1 \leq i \leq n$, with $\left\{e_{i}\right\}_{i=1}^{n}$ the standard basis of $\mathbf{R}^{n}$. Let $u \in C^{2}(\bar{\Omega})$ be a solution of (1) such that $u(x)>0, x \in \Omega$ and there exists $x \in \Omega$ such that $u(x)>s_{1}$. Let $r \in\left(s_{1}, s_{2}\right)$ be defined by $\int_{s_{0}}^{r} f(s) d s=0$. Then $\|u\|_{0} \geq r$.

ProOF. It follows from Theorem 1 that $\|u\|_{0}=u(0)$, and that $u(x)<\|u\|_{0}, x \neq$ 0. Furthermore, it follows from the result of [6] (that $\gamma_{i}=e_{i}, 1 \leq i \leq n$, is important here) that the level sets $\Omega_{c}=\{x: u(x) \geq c\}$ are starshaped with respect to 0 . We now recall an identity of Rellich (see $[\mathbf{2}$, p. 201]):

$$
\begin{aligned}
& \frac{1}{2} \int_{\partial \Omega_{c}} \nu \cdot x\left(\frac{\partial u}{\partial \nu}\right)^{2} d S+F(c) \int_{\partial \Omega_{c}} \nu \cdot x d S \\
& \quad=\frac{2-n}{2} \int_{\Omega_{c}}|\nabla u|^{2} d x+n \int_{\Omega_{c}} F(u) d x
\end{aligned}
$$

where $\nu$ is the unit outward normal vector field to $\Omega_{c}$ and $F(t)=\int_{t^{*}}^{t} f(\tau) d \tau$, where $t^{*}$ is fixed.

Choosing $t^{*}=s_{0}$ and $c=s_{0}<\|u\|_{0}$, we obtain that

$$
n \int_{\Omega_{s_{0}}} F(u) d x=\frac{1}{2} \int_{\partial \Omega_{s_{0}}} \nu \cdot x\left(\frac{\partial u}{\partial \nu}\right)^{2} d S+\frac{n-2}{2} \int_{\Omega_{s_{0}}}|\nabla u|^{2} d x .
$$

Since $\Omega_{s_{0}}$ is starshaped with respect to 0 , i.e. $\nu \cdot x \geq 0, x \in \partial \Omega_{s_{0}}$, it follows that $\int_{\Omega_{s_{0}}} F(u) d x \geq 0$, hence there exists $x \in \Omega_{s_{0}}$ such that

$$
F(u(x))=\int_{s_{0}}^{u(x)} f(x) d x \geq 0
$$

i.e. $u(x) \geq r$.

If it is the case that $\Omega$ is convex we obtain the same result using a different argument. (Whether or not the level sets $\Omega_{c}$ are starshaped in this more general setting is unknown to us at this point.)

THEOREM 3. Let $\Omega$ be convex. Let $u \in C^{3}(\Omega) \cap C^{2}(\bar{\Omega})$ be a solution of $(1)$ such that $u(x)>0, x \in \Omega$ and there exists $x \in \Omega$ such that $u(x)>s_{1}$. Let $r \in\left(s_{1}, s_{2}\right)$ be defined by $\int_{s_{0}}^{r} f(s) d s=0$. Then $\|u\|_{0} \geq r$.

PROOF. Let $u$ be a solution of (1) satisfying the above hypotheses.

Let

$$
P(x)=\frac{1}{2}|\nabla u(x)|^{2}+\int_{s_{0}}^{u(x)} f(\tau) d \tau,
$$

then since $\Omega$ is convex, it follows from results of Payne (see $[9$, p. 77]) that $P$ assumes its maximum where $\nabla u=0$. Thus we have that

$$
\max P(x)=P\left(x_{0}\right)=\int_{s_{0}}^{u\left(x_{0}\right)} f(\tau) d \tau \geq 0
$$


since $\left\{x: u(x)=s_{0}\right\} \neq \varnothing$. Hence either $u\left(x_{0}\right) \geq r$ or $u\left(x_{0}\right)=s_{0}$. If $u\left(x_{0}\right)=s_{0}$, then $P\left(x_{0}\right)=0$ and thus $P(x)=0$ for all $x$ such that $u(x)=s_{0}$, i.e. $|\nabla u(x)|=0$ for all such $x$. We now apply Rellich's identity (4) to $\Omega_{s_{0}}$ from which it follows that

$$
\frac{n-2}{2} \int_{\Omega_{s_{0}}}|\nabla u|^{2} d x=n \int_{\Omega_{s_{0}}} F(u) d x
$$

Since $n \geq 2, \int_{\Omega_{s_{0}}} F(u) d x \geq 0$, so there exists $x \in \Omega_{s_{0}}$ such that $\int_{s_{0}}^{u(x)} f(\tau) d \tau \geq 0$. But $x \in \Omega_{s_{0}}$ implies $u(x)>s_{0}$ and thus $u(x) \geq r$. Therefore $u_{\max } \geq r$.

\section{REFERENCES}

1. A. Ambrosetti and P. Hess, Positive solutions of asymptotically linear eigenvalue problems, J. Math. Anal. Appl. 73 (1980), 411-422.

2. C. Bandle, Isoperimetric inequalities and applications, Pitman, Boston, Mass., 1980.

3. K. J. Brown and H. Budin, On the existence of positive solutions for a class of semilinear elliptic boundary value problems, SIAM J. Math. Anal. 10 (1979), 875-883.

4. B. Gidas, W. M. Ni and L. Nirenberg, Symmetry and related properties via the maximum principle, Comm. Math. Phys. 68 (1979), 209-243.

5. P. Hess, On multiple positive solutions of nonlinear elliptic eigenvalue problems, Comm. Partial Differential Equations 6 (1981), 951-961.

6. B. Kawohl, A geometric property of level sets of solutions to semilinear elliptic Dirichlet problems, Appl. Analysis 16 (1983), 229-233.

7. H. O. Peitgen, D. Saupe and K. Schmitt, Nonlinear elliptic boundary value problem versus their finite difference approximations: Numerically irrelevant solutions, J. Reine Angew. Math. 322 (1981), 74117.

8. H. O. Peitgen and K. Schmitt, Global topological perturbations of nonlinear elliptic eigenvalue problems, Math. Appl. Sci. 5 (1983), 376-388.

9. R. Sperb, Maximum principles and their applications, Academic Press, New York, 1981.

Department of MAThematics and Computer SCIENCE, UNiVERsity of Miami, CORAL Gables, Florida 33124

Department of Mathematics, University of Utah, SAlt LAKE City, Utah 84112 